\title{
Fast magnetosonic waves driven by gravitational waves
}

\author{
D. Papadopoulos ${ }^{1}$, N. Stergioulas ${ }^{1}$, L. Vlahos ${ }^{1}$, and J. Kuijpers ${ }^{2,3}$ \\ 1 Department of Physics, Aristoteleion University of Thessaloniki, 54006 Thessaloniki, Greece \\ 2 Department of Astrophysics, University of Nijmegen, PO Box 9010, 6500 GL Nijmegen, The Netherlands \\ 3 Astronomical Institute, Utrecht University, PO Box 80 000, 3508 TA Utrecht, The Netherlands
}

Received 18 April 2001 / Accepted 7 June 2001

\begin{abstract}
The propagation of a gravitational wave (GW) through a magnetized plasma is considered. In particular, we study the excitation of fast magnetosonic waves (MSW) by a gravitational wave, using the linearized generalrelativistic hydromagnetic equations. We derive the dispersion relation for the plasma, treating the gravitational wave as a perturbation in a Minkowski background space-time. We show that the presence of gravitational waves will drive magnetosonic waves in the plasma and discuss the potential astrophysical implications.
\end{abstract}

Key words. gravitational waves - MHD waves - compact objects: pulsars; magnetars; GRBs.

\section{Introduction}

It is well known that gravitational waves (GWs) can interact with electromagnetic waves. The scattering of electromagnetic waves by time dependent GWs has also been considered by several authors (DeWitt \& Breheme 1960; Cooperstock 1968; Chestrs 1973; Denisov 1978; Grischuk \& Polnarev 1980; Macdonald \& Thorne 1982; Machedo \& Nelson 1983; Demianski 1983; Marklund et al. 2000). It has been shown that the coupling is mainly due to the generation of electric currents inside the plasma by the perturbation of the charged particle trajectories by the GW. Several studies used the kinetic theory formulation to study the coupling of GWs with electromagnetic waves in a magnetized vacuum (Gertsenshtein 1962; Zeldovich 1974) or near a charged black hole (Gerlach 1974). The first attempt to study the propagation of GWs in a magnetized plasma using the kinetic theory was by Macedo \& Nelson (1983). They studied the coupling of GWs propagating parallel and perpendicular to the external magnetic field with high-frequency electromagnetic modes. The coupling of the gravitational with the high-frequency electromagnetic modes propagating along the magnetic field was weak. This is due to the fact that the GW propagating along the direction of the undisturbed magnetic field does not generate currents in the plasma. The coupling is substantially higher for ordinary and extraordinary waves propagating perpendicular to the magnetic field but the energy transfer of gravitational energy to electromagnetic energy in the interstellar space is extremely weak.

Send offprint requests to: D. Papadopoulos,

e-mail: papadop@astro.auth.gr
Brodin \& Marklund (1999) discuss the parametric excitation of plasma waves in the presence of gravitational radiation. They assumed that the GW was a monochromatic wave and estimated, using EinsteinMaxwell-Vlasov's equations, the growth of Langmuir and electromagnetic waves in a cold unmagnetized plasma. Marklund et al. (2000) used the $1+3$ orthonormal frame formalism (Ellis \& van Elst 1998) to study the linear interaction of a GW inside a inhomogeneous magnetized plasma. They showed that, close to a strong gravitational source, the electric fields associated with the electrostatic waves can reach appreciable levels. The direct escape of plasma radiation is not possible without a nonlinear conversion (see Brodin et al. 2000). Recently Servin et al. (2000) discuss the non-linear parametric excitation of Alfvén waves by a strong monochromatic GW. A nonlinear study of shock waves in a highly magnetized plasma, driven by GWs has been presented by Ignat'ev (1997).

The non-linear interaction of a monochromatic GW with charged particles and their cyclotron damping inside a plasma has also been analyzed in detail (see Esposito 1971; Polnarev 1972; Asseo et al. 1976; Papadopoulos \& Esposito 1985; Varvoglis \& Papadopoulos 1992; Servin et al. 2001)

Papadopoulos \& Esposito (1982) derived the exact equations for a finite amplitude MHD wave propagating inside the plasma, within the framework of general relativity.

In this article we study, for the first time, the excitation of magnetosonic waves from a $\mathrm{GW}$ propagating inside a uniform, strongly magnetized plasma (where the Alfvén speed approaches the speed of light). In Sect. 2 we 
outline the basic equations, in Sect. 3 we derive the general dispersion relation for the coupling of the GWs with the MHD waves and in Sect. 4 the dispersion relation for the coupling of the gravitational with ambient magnetosonic waves. In Sect. 5 we discuss our results and their potential astrophysical implications, while Sect. 6 summarizes our conclusions.

\section{Basic equations}

The equations for finite amplitude wave propagation in an ideal hydromagnetic plasma in the general relativistic framework are (see Papadopoulos \& Esposito 1982)

$$
\begin{aligned}
(\epsilon & \left.-\frac{H^{2}}{2}\right)_{; a b} u^{a} u^{b}=h^{a b}\left(p+\frac{H^{2}}{2}\right)_{; a b}+2\left(H^{2} \Theta\right) \\
& -\left(H^{a} H^{b}\right)_{; a b}+2 x\left(\frac{2 \Theta^{2}}{3}+\sigma^{2}-\omega^{2}-\dot{u}^{a} \dot{u}_{a}\right) \\
& +\frac{x}{2}\left(\rho+3 p+H^{2}\right)+2 \dot{u}_{a}\left(H^{a} H^{b}\right)_{; b}+\left(H^{2}\right)_{; a} \dot{u}^{a}
\end{aligned}
$$

Maxwell's equations take on the compact form

$$
\dot{H}^{a}=\left(\sigma_{b}^{a}+\omega_{b}^{a}-\frac{2}{3} \delta_{b}^{a} \Theta\right) H^{b}+\frac{1}{\epsilon+p} p_{; b} H^{b} u^{a}
$$

and the equation of motion is

$$
\dot{x} u^{a}+x \dot{u}^{a}+x \Theta u^{a}+\left(p+\frac{H^{2}}{2}\right)_{; b} g^{a b}-\left(H^{a} H^{b}\right)_{; b}=0(3)
$$

where $u^{a}$ is the fluid velocity, $\epsilon$ is the energy density, $\rho$ the rest mass density, $p$ the pressure of the fluid, $H^{a}=\left(0, H^{1}, H^{2}, H^{3}\right)$ is the prevailing magnetic field, $\dot{u}^{a}=u_{; c}^{a} u^{c}, x=\epsilon+p+H^{2}, h^{a b}=g^{a b}+u^{a} u^{b}, \sigma_{i j}$ is the shear, $\omega_{i j}$ is an antisymmetric tensor, corresponding to the rotation of the fluid and $\Theta$ is the expansion of the fluid. In the equations we have assumed $c=1, \kappa=8 \pi G / c^{4}=1$ and we have put $H=B \rightarrow \sqrt{4 \pi} H$ (from Gaussian units), where $\kappa$ is the coupling constant in Einstein's field equations.

We perturb the Eqs. (1)-(2) and keep only linear terms in the perturbed quantities (see Eqs. (47)-(49) in Papadopoulos \& Esposito 1982), and use the metric given by $g_{\mu \nu}=\eta_{\mu \nu}+h_{\mu \nu}$ or

$\mathrm{d} s^{2}=-c^{2} \mathrm{~d} t^{2}+(1+h) \mathrm{d} x^{2}+(1-h) \mathrm{d} y^{2}+\mathrm{d} z^{2}$

where $h=h(t, z)$ represents the perturbation of the flat space by the GW.

In the following section we will search for the coupling of the normal modes of the magnetized plasma with the GW. It is important to notice that both gravitational and MHD waves follow similar dispersion relations, in the asymptotic limit when the relativistic Alfvén speed approaches the speed of light (see also Landau \& Lifshitz 1975).

\section{The perturbed equations}

In this section we will perturb Eqs. (1)-(2) assuming that $\delta g_{a b} \neq 0$. Perturbing Eq. (1) we find

$\delta\left[\left(\epsilon-\frac{H^{2}}{2}\right)_{; a b}\right] u^{a} u^{b}-\delta \Gamma_{a b}^{k}\left(\epsilon-\frac{H^{2}}{2}\right)_{, k} u^{a} u^{b}$

$+\left(\epsilon-\frac{H^{2}}{2}\right)_{; a b}\left(u^{a} \delta u^{b}+u^{b} \delta u^{a}\right)+\left(\delta \epsilon-H^{c} \delta H_{c}\right)_{; a b} u^{a} u^{b}$

$=\delta g^{a b} p_{, a b}^{*}+\left(u^{a} \delta u^{b}+u^{b} \delta u^{a}\right) p_{a b}^{*}+h^{a b}\left(\delta p_{, a b}^{*}-\delta \Gamma_{a b}^{k} p_{, k}^{*}\right)$

$+2 \delta\left[\left(H^{2}\right)_{, c} \Theta+H^{2} \Theta_{, c}\right] u^{c}$

$-H^{b}\left[2 \delta\left(H_{, a b}^{a}\right)+H^{k} \delta\left(\Gamma_{a k, b}^{a}\right)+2 \delta \Gamma_{a k}^{a} H_{, b}^{k}\right]$

$-2 H_{, a b}^{a} \delta H^{b}-2 \delta \Gamma_{a l}^{a} H^{l} H_{, b}^{b}-\delta\left(H_{, b}^{a}\right) H_{, a}^{b}-H_{, b}^{a} \delta\left(H_{, a}^{b}\right)$

$-\delta \Gamma_{b l}^{a} H^{l} H_{, a}^{b}-H^{a} H^{c} \delta \Gamma_{a c, b}^{b}-\delta \Gamma_{a c}^{b} H_{, b}^{c} H^{a}$

$+2 x\left[\sigma \delta \sigma-u^{c} u^{d}\left(\delta u_{, c}^{a} u_{a, d}+u_{, c}^{a} \delta u_{a, d}\right.\right.$

$\left.\left.+\delta \Gamma_{c l}^{a} u^{l} u_{a, d}-u_{, c}^{a} u_{m} \delta \Gamma_{a d}^{m}\right)-\left(u^{d} \delta u^{c}+u^{c} \delta u^{d}\right) u_{, c}^{a} u_{a, d}\right]$

$+\frac{1}{2}\left(\epsilon+3 p+H^{2}\right)\left(\delta \epsilon+\delta p+\delta H^{2}\right)+\frac{1}{2} x\left(\delta \epsilon+3 \delta p+\delta H^{2}\right)$

$+\left[2 \delta u^{c} u_{a, c}+2 u^{c}\left(\delta u_{a, c}-\delta \Gamma_{a c}^{m} u_{m}\right)\right]\left(H^{a} \delta H_{b}+H^{b} \delta H^{a}\right)$

$+\left(H^{2}\right)_{; a}\left(\delta u^{a}\right)_{; c} u^{c}$

$+\left(H^{2}\right)_{, a} u_{, c}^{a} \delta u^{c}+\left(H^{2}\right)_{, a} u^{c}\left(\delta u_{, c}^{a}+\delta \Gamma_{c m}^{a} u_{m}\right)$.

The perturbed Maxwell's equations are

$\delta H_{, 0}^{b}+\delta \Gamma_{0 l} H^{l}=-\delta \sigma_{a}^{b} H^{a}+\frac{2}{3} H^{b} \delta \Theta-\frac{u^{b}}{\epsilon+p} \delta p_{, c} H^{c}$

and from Eq. (3) we find the perturbed equations of motion

$$
\begin{aligned}
& -\left(\epsilon+p+H^{2}\right) \delta u_{, 0}^{c}=h_{a}^{c}\left[H^{b} \delta H_{, b}^{a}+\delta \Gamma_{b l}^{a} H^{b} H^{l}\right. \\
& \left.+H^{a} \delta H_{, b}^{b}+\delta \Gamma_{b m}^{b} H^{m} H^{a}\right]-\delta h^{c b} p_{, b}^{*}-h^{c b} \delta p_{, b}^{*}
\end{aligned}
$$

where $p^{*}=p+\frac{H^{2}}{2}$. The non-zero Christoffel symbols $\Gamma_{\mu \nu}^{\alpha}$, for the metric (4), are estimated easily $\Gamma_{11}^{0}=-\Gamma_{22}^{0}=$ $-\frac{h, 0}{2}, \Gamma_{01}^{0}=\Gamma_{10}^{0}=\frac{h, 0}{2(1+h)}, \Gamma_{13}^{1}=\Gamma_{31}^{1}=\frac{h, 3}{2(1+h)}, \Gamma_{02}^{2}=$ $\Gamma_{20}^{2}=-\frac{h, 0}{2(1-h)}, \Gamma_{23}^{2}=\Gamma_{32}^{2}=-\frac{h, 3}{2(1-h)}, \Gamma_{11}^{3}=\Gamma_{22}^{3}=\frac{h, 3}{2}$. We assume that $u^{a}=(-1,0,0,0), \rho=\rho_{0}=$ const. $H^{a}=\left(0, H^{1}, 0, H^{3}\right)=$ const., $\delta H^{a}=\left(0, \delta H^{1}, \delta H^{2}, 0\right)$, $\delta u^{a}=\left(\delta u^{0}, \delta u^{1}, \delta u^{2}, \delta u^{3}\right)$ and $k^{a}=(0,0,0, k)$ and a plane wave behaviour for the perturbed quantities $\delta \epsilon, \delta p, \delta u^{a}$ and $\delta H^{a} \sim \mathrm{e}^{i(k z-n t)}$ and the GW $h \sim \mathrm{e}^{i\left(k_{\mathrm{g}} z-n_{\mathrm{g}} t\right)}$. These simplifications yield a general dispersion relation for the coupling of GW with MHD waves.

$\delta \epsilon\left\{D_{0}\left(-n^{2}-2 n^{2} u_{\mathrm{A}}^{2}\right)+\left(k c_{\mathrm{s}}\right)^{2} D_{0}\right.$

$-\frac{1}{2}\left(1+c_{\mathrm{s}}^{2}\right)\left(\epsilon+3 p+H^{2}\right) D_{0}$

$-\frac{1}{2}\left(1+3 c_{\mathrm{s}}^{2}\right)\left(\epsilon+p+H^{2}\right) D_{0}$

$+\left[n^{2}+k^{2}-2 n^{2} u_{\mathrm{A}}^{2}-2\left(\epsilon+2 p+H^{2}\right)\right]\left(\lambda_{1} H^{1}+\lambda_{2} H^{1} c_{\mathrm{s}}^{2}\right)$

$\left.+\left[n^{2}-k^{2}-2 n^{2} u_{\mathrm{A}}^{2}-2\left(\epsilon+2 p+H^{2}\right)\right]\left(\mu_{1} H^{3}+\mu_{2} H^{3} c_{\mathrm{s}}^{2}\right)\right\}$

$=h\left\{D_{0}\left(H^{1}\right)^{2}\left(\epsilon+2 p+H^{2}-k_{\mathrm{g}}^{2}\right)-\lambda_{3} H^{1}-\mu_{3} H^{3}\right\}$ 
where

$$
\begin{aligned}
& \delta H^{1}=\frac{1}{D_{0}}\left(\lambda_{1} \delta \epsilon+\lambda_{2} \delta p+\lambda_{3} h\right) \\
& \delta H^{3}=\frac{1}{D_{0}}\left(\mu_{1} \delta \epsilon+\mu_{2} \delta p+\mu_{3} h\right)
\end{aligned}
$$

with

$$
\begin{aligned}
\lambda_{1}= & -\frac{H^{1}}{x}\left(1-\frac{k^{2} u_{\mathrm{A}}^{2}}{2 n^{2}}\right), \lambda_{2}=\frac{\left(H^{1} H^{3}\right)}{x} \frac{H^{3}}{x} \frac{k^{2}}{n^{2}}\left(1+\frac{k^{2}}{2 n^{2}}\right), \\
\lambda_{3}= & \left(-\lambda_{1}+\lambda_{2}\right)\left[\frac{k^{2}\left(H^{3}\right)^{2}}{2 n^{2}}-\frac{n_{\mathrm{g}} x}{n}\right] \\
& +\frac{\left(H^{1}\right)}{x} \frac{\left(H^{1} H^{3}\right)^{2}}{x} \frac{k k_{\mathrm{g}}}{2 n^{2}}\left(1+\frac{k^{2}}{2 n^{2}}\right), \\
\mu_{1}= & -\frac{H^{3}}{x}\left(1-\frac{k^{2} u_{\mathrm{A}}^{2}}{2 n^{2}}\right), \\
\mu_{2}= & \frac{H^{3}}{x} \frac{k^{2}}{n^{2}}\left[1-\frac{\left(H^{1}\right)^{2}}{x}-\frac{k^{2}}{2 n^{2}} \frac{\left(H^{3}\right)^{2}}{x}\right], \\
\mu_{3}= & \frac{\left(H^{1} H^{3}\right)}{x} \frac{k k_{\mathrm{g}}}{2 n^{2}} H^{1}\left[1-\frac{\left(H^{1}\right)^{2}}{x}\right]-\frac{k^{3} k_{\mathrm{g}}}{4 n^{4}} \frac{\left(H^{1} H^{3}\right)^{2}}{x^{2}} H^{3} \\
& -\frac{H^{1}}{x} \frac{\left(H^{1} H^{3}\right)}{x}\left[\frac{k^{2}\left(H^{3}\right)^{2}}{2 n^{2}}-\frac{n_{\mathrm{g}} x}{n}\right]\left(1-\frac{k^{2}}{2 n^{2}}\right),
\end{aligned}
$$

and

$$
\begin{aligned}
-D_{0}= & 1-u_{A}^{2}-\frac{k^{2} u_{\mathrm{A}}^{2}}{2 n^{2}} \\
- & \frac{k^{2}}{n^{2}} \frac{\left(H^{3}\right)^{2}}{x}\left[1-\frac{k^{2}}{2 n^{2}} \frac{\left(H^{3}\right)^{2}}{x}-\frac{\left(H^{1}\right)^{2}}{x}\right] \\
& +\frac{k^{2} u_{\mathrm{A}}^{2}}{2 n^{2}} \frac{\left(H^{1}\right)^{2}}{x}+\frac{k^{4} u_{\mathrm{A}}^{2}}{2 n^{4}} \frac{\left(H^{3}\right)^{2}}{x} .
\end{aligned}
$$

Above, $u_{\mathrm{A}}^{2}=v_{\mathrm{A}}^{2} /\left(1+v_{\mathrm{A}}^{2}\right)$ is a relativistic generalization of the classical Alfvén speed $v_{\mathrm{A}}=H^{2} /\left(4 \pi \epsilon / c^{2}\right)$ and $c_{\mathrm{s}}=\sqrt{\delta p / \delta \epsilon}$ is the speed of sound. In passing we note that the non-relativistic Alfvén speed $v_{\mathrm{A}}$ generalizes to the relativistic Alfvén speed $u_{\mathrm{A}}$ if one does not neglect the displacement current in Maxwell's equation, in MHD theory.

\section{Magnetosonic waves}

We are interested in investigating the dispersion relation for waves that correspond to the magnetosonic mode $\left(H^{1} \neq 0, H^{3}=0\right)$. This is motivated by the fact that the coupling of MHD waves with GWs is proportional to $\left(H^{1}\right)^{2}$. Thus, gravitational waves in the $z$-direction cannot couple to pure Alfvén waves $\left(H^{1}=0, H^{3} \neq 0\right)$. The general relations between the perturbed quantities, derived above, simplify when we assume that $H^{3}=0$ (setting $H^{1}=H$ from now on)

$$
\begin{aligned}
-n^{2} \delta \epsilon & -2 n^{2} \frac{H^{2}}{x} \delta \epsilon+k^{2} \delta p-\frac{1}{2}(\delta \epsilon+\delta p)\left(\epsilon+3 p+H^{2}\right) \\
& -\frac{1}{2}(\delta \epsilon+3 \delta p)\left(\epsilon+p+H^{2}\right)+(H \delta H)\left[n^{2}+k^{2}\right. \\
& \left.-2 n^{2} \frac{H^{2}}{x}-2\left(\epsilon+2 p+H^{2}\right)\right] \\
& =h\left(H^{2}\right)\left[\left(\epsilon+2 p+H^{2}\right)-k_{\mathrm{g}}^{2}\right]
\end{aligned}
$$

where, from Maxwell's Eq. (6) we obtain

$$
(H \delta H)=\frac{H^{2}}{1-u_{\mathrm{A}}^{2}}\left[\frac{\delta \epsilon}{x}-h \frac{n_{\mathrm{g}}}{n}\right] .
$$

From here on, we will write our equations in ordinary units again. We combine Eqs. (9)-(10) to obtain

$$
\begin{aligned}
& \delta \epsilon\left\{-n^{2}+\left(k c_{\mathrm{s}}\right)^{2}+k^{2} u_{\mathrm{A}}^{2}-\left(k c_{\mathrm{s}}\right)^{2} \frac{u_{\mathrm{A}}^{2}}{c^{2}}\right. \\
& -2 G\left(4 \pi \rho+\frac{H^{2}}{c^{2}}\right)\left(1+2 \frac{c_{\mathrm{s}}^{2}}{c^{2}}\right)\left(1-\frac{u_{\mathrm{A}}^{2}}{c^{2}}\right) \\
& \left.-4 G \frac{u_{\mathrm{A}}^{2}}{c^{2}}\left(4 \pi \rho+\frac{H^{2}}{c^{2}}\right)\right\} \\
& =h \frac{H^{2}}{4 \pi}\left\{\frac{n_{\mathrm{g}}}{n}\left[n^{2}\left(1-2 \frac{u_{\mathrm{A}}^{2}}{c^{2}}\right)+c^{2} k^{2}-4 G\left(4 \pi \rho+\frac{H^{2}}{c^{2}}\right)\right]\right. \\
& \left.\quad+\left(1-\frac{u_{\mathrm{A}}^{2}}{c^{2}}\right)\left(4 \pi G \rho+\frac{G H^{2}}{c^{2}}-k_{\mathrm{g}}^{2} c^{2}\right)\right\}
\end{aligned}
$$

where $H^{2}=H_{a} H^{a}=H_{1} H^{1}$ and we have set $p=0$ and $\rho=\epsilon / c^{2}$.

We retain the dominant terms that yield a pure magnetosonic wave in a strongly magnetized, cold and tenuous plasma $\left(c_{\mathrm{s}} \ll u_{\mathrm{A}}\right)$ in the Newtonian limit. This is equivalent to setting $1 / c^{2} \rightarrow 0$ (except for the $u_{\mathrm{A}}^{2} / c^{2}$ terms) and $G \rho \rightarrow 0, c_{\mathrm{s}} \rightarrow 0$ and one obtains

$$
\begin{aligned}
\left(-n^{2}+k^{2} u_{\mathrm{A}}^{2}\right) \delta \epsilon= & h \frac{H^{2}}{4 \pi}\left\{\frac{n_{\mathrm{g}}}{n}\left[n^{2}\left(1-2 \frac{u_{\mathrm{A}}^{2}}{c^{2}}\right)\right]\right. \\
& \left.+k^{2} c^{2}-\left(1-\frac{u_{\mathrm{A}}^{2}}{c^{2}}\right) k_{\mathrm{g}}^{2} c^{2}\right\} .
\end{aligned}
$$

In the absence of a $\mathrm{GW}(h=0)$, the dispersion relation (12) becomes

$n_{r}^{2} \simeq k^{2} u_{\mathrm{A}}^{2}$.

If one assumes that the frequency of the driving GW coincides with the frequency of the produced MSW, i.e. $n=n_{\mathrm{g}}=k_{\mathrm{g}} c$, then the dispersion relation becomes

$\left(-n_{\mathrm{g}}^{2}+k^{2} u_{\mathrm{A}}^{2}\right) \delta \epsilon=h \frac{H^{2}}{4 \pi}\left(-n_{\mathrm{g}}^{2}+k^{2} c^{2}\right)$,

or

$\left(\frac{\partial^{2}}{\partial t^{2}}-u_{\mathrm{A}}^{2} \frac{\partial^{2}}{\partial z^{2}}\right) \delta \epsilon=h \frac{H^{2}}{4 \pi}\left(-n_{\mathrm{g}}^{2}+k^{2} c^{2}\right)$, 
i.e. the GW acts as an external parametric oscillator, driving the MSW. Notice also that, in the absence of a plasma one obtains the dispersion relation for GWs propagating in the vacuum. The two wave numbers differ by an amount $\Delta k$, i.e. $k=k_{\mathrm{g}}+\Delta k$, which, in general, is complex, $\Delta k=\Delta k_{\mathrm{R}}+i \Delta k_{\mathrm{I}}$. If we denote

$$
R=\left(\frac{h}{\delta \epsilon}\right)_{0} \frac{H^{2}}{4 \pi}
$$

(where a subscript 0 denotes amplitude), then (14) yields a system of two equations, for $\Delta k_{\mathrm{R}}$ and $\Delta k_{\mathrm{I}}$. Dividing the two resulting equations, one obtains

$\frac{-n_{\mathrm{g}}^{2}+\left[k_{\mathrm{g}}^{2}+2 k_{\mathrm{g}} \Delta k_{\mathrm{R}}+\Delta k_{\mathrm{R}}^{2}\right] u_{\mathrm{A}}^{2}}{2 k_{\mathrm{g}} \Delta k_{\mathrm{I}} u_{\mathrm{A}}^{2}}=\frac{A \cos \phi+B \sin \phi}{A \sin \phi+B \cos \phi}$

where

$A=\left[2 k_{\mathrm{g}} \Delta k_{\mathrm{R}}+\Delta k_{\mathrm{R}}^{2}\right] c^{2}$,

$B=2 k_{\mathrm{g}} \Delta k_{\mathrm{I}} c^{2}$

and

$\phi=\Delta k_{\mathrm{R}} z$

When $\left|\Delta k_{\mathrm{I}}\right| \ll\left|\Delta k_{\mathrm{R}}\right| \Rightarrow|B / A| \ll 1$ and since $u_{\mathrm{A}} \simeq c$ in a strongly magnetized, tenuous plasma, we arrive at an expression for $\Delta k_{\mathrm{I}}$

$\Delta k_{\mathrm{I}} \simeq-\frac{3 k_{\mathrm{g}}}{2}\left(1-\frac{u_{\mathrm{A}}}{c}\right) \tan \Delta k_{\mathrm{R}} z$.

The real part of the wavenumber difference is approximately

$\Delta k_{\mathrm{R}}=k-k_{\mathrm{g}}=k_{\mathrm{g}}\left(\frac{c}{u_{\mathrm{A}}}-1\right)$,

thus, the two waves can linearly interact coherently over a maximum distance of

$L \approx \frac{\lambda_{g}}{2 \pi\left(1-u_{a} / c\right)}$

in terms of the GW wavelength $\lambda_{\mathrm{g}}$.

For the plasma around a magnetized compact object, $B^{2} / \epsilon \sim 10^{2}-10^{4}$ (where $\epsilon$ is dominated by the kinetic energy of the plasma particles). For such a plasma, the interaction length can be up to several thousand times larger than $\lambda_{\mathrm{g}}$, A more stringent limit on the interaction length will probably be set but factors such as the nonuniformity of the magnetic field and the plasma density with distance from the source.

Assuming also a small imaginary part for the frequency and substituting $n=n_{r}+i \gamma$ in Eq. (14), developing $-n^{2}+$ $k^{2} u_{\mathrm{A}}^{2}=\left(n-n_{r}+i \gamma\right)\left(-2 n_{r}\right)$, and using the Plemelj formula $\left(\frac{\lim }{\gamma \rightarrow 0^{+}}(x+i \gamma)^{-1}=P(1 / x)-i \pi \delta(x)\right)$ we finally arrive at the spatial dependence of the amplitude of the energy density

$\delta \epsilon_{0} \approx \frac{i}{4} \delta\left(n-n_{g}\right) h_{0} H^{2} c \Delta k \mathrm{e}^{-i(\Delta k) z}$, where $\delta \epsilon=\delta \epsilon_{0} \mathrm{e}^{i(n t-k z)}, \delta H=\delta H_{0} \mathrm{e}^{i(n t-k z)}$ and $h=$ $h_{0} \mathrm{e}^{i\left(n_{\mathrm{g}} t-k_{\mathrm{g}} z\right)}$.

The magnetic field amplitude is estimated with the use of the perturbed Maxwell's Eq. (6) and with the aid of Eq. (10)

$\delta H_{0} \approx \frac{i}{2} \delta\left(n-n_{\mathrm{g}}\right) h_{0} H c \Delta k \mathrm{e}^{-i \Delta k z}$.

We note that, the amplitude of the fast magnetosonic wave is proportional to the GW amplitude. In a tenuous and hot plasma, $u_{\mathrm{A}} \rightarrow c$, so that the mismatch $\Delta k \ll k$ and our treatment applies. As a result the amplitude of the MSW grows after having traveled a distance equal to a half beat between the wave numbers, and would decline again further out in the absence of dissipation. In a relativistic electron positron plasma the latter is, however, unlikely and some observational effects can be expected.

\section{Discussion}

\subsection{Interpretation}

We have found that, to first order, a GW, propagating along the magnetic field in a uniform and ideal plasma, does not couple with an Alfvén wave (AW) whereas a GW, propagating across the magnetic field, does couple with a MSW. This can be easily be understood as follows.

In Fig. 1 we have sketched the effect of a largeamplitude GW traveling along an, initially uniform, magnetic field embedded in an ideal plasma. At first sight it is surprising that the GW would not be able to excite an AW as the perturbed magnetic field shows clear distortions which in an ordinary magnetoplasma would be expected to generate AWs. However, the point is that the energy of an AW with amplitude $\delta H^{1}$ is proportional to $\left(\delta H^{1}\right)^{2}$ whereas, for a MSW, the magnetic part of the wave energy density is proportional to $H^{1} \delta H^{1}$. As a result, the energy in the AW is of higher order in the perturbation as compared to the magnetosonic case. Therefore, our first order calculation should not lead to the excitation of AWs, as indeed we have found. On the other hand, we do expect such a coupling to show up in a higher-order calculation, and such a coupling will be relevant in case the GW is non-linear.

In Fig. 2 we have sketched the effect of a GW traveling at right angles to an initially uniform magnetic field frozen into an ideal plasma. Clearly, the field is periodically compressed by the GW although the differential motions induced by the GW are divergence-free! At the same time, the ambient uniform magnetic field remains uniform and straight and does not become bent. As a result, no AW excitation is expected to occur by the GW even to higher order.

Further, our result that the coupling between a GW and MSWs vanishes as soon as the dispersion relations for both wave modes coincide exactly, can be understood as follows. An effective transfer of energy from one wave mode to the other either requires that there is some dissipation (Weber 1960; Esposito 1971) or, alternatively, that 


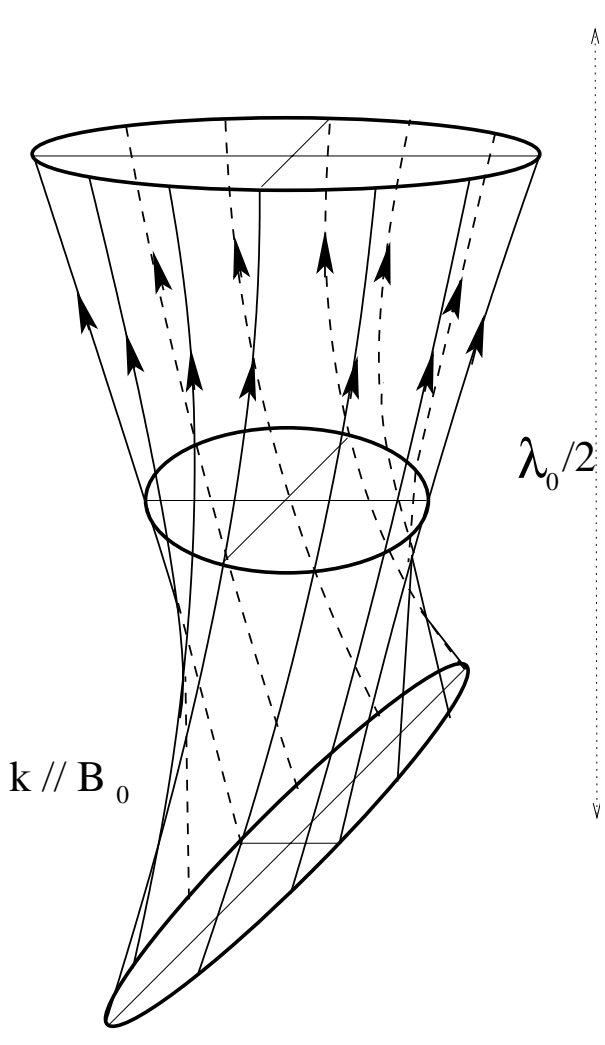

Fig. 1. Sketch of the effect of a large-amplitude GW traveling parallel to a uniform magnetic field frozen into an ambient ideal plasma, $\boldsymbol{k} \| \boldsymbol{B}_{\mathbf{0}}$ at an arbitrary time. Here the vertical direction coincides with the direction of wave propagation and of the background magnetic field.

the signal speeds of both modes differ. If the plasma is both ideal and the group speeds of the wave modes coincide, there is no time lag during which some of the perturbed kinetic/magnetic plasma energy established by the GW can be fed into a magnetosonic wave. In a very general sense, the changes brought about by the GW in the magnetoplasma are completely adiabatic and the periodic changes in the plasma completely reversible. All free energy in the magnetic field reverts back into the GW as it leaves the plasma again.

\subsection{Applications and perspective}

A number of papers exist which discuss the interaction of MSWs with a plasma, e.g. coherent MSW-particle interactions, cascading of long wave-length MSWs to short wavelengths and subsequent particle acceleration from turbulent diffusion (see Dermer et al. 1996), and finally the interaction of a relativistic magnetosonic shock wave with a positron-electron plasma (Hoshino et al. 1992). Thus, if a magnetosonic wave is driven by a GW, then it has the potential of producing additional interesting phenomena. A more detailed understanding of the linear and nonlinear evolution of the excited MSWs under the conditions of the particular GW source is needed. Clearly, it is beyond the

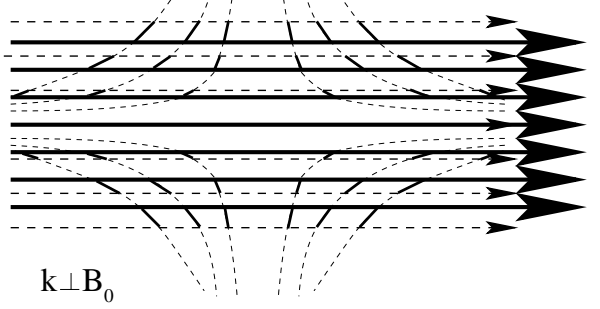

Fig. 2. Sketch of the effect of a GW traveling perpendicular to a uniform magnetic field frozen into an ideal plasma. The tidal motion of the GW is indicated with curved dashed lines. The drawn and, respectively, dashed arrowed lines are the magnetic field lines half a cycle apart. Their individual relative displacements are shown by the bold sections along the tidal curves. Note that the field lines remain straight under the action of the GW.

scope of the present study to model the acceleration and radiation properties of a GW-driven MSW in the vicinity of a GW-source but we want to mention briefly two specific applications which have been the motivation for us to undertake this study: GWs excited by star quakes or rotational instabilities in neutron stars (see Andersson et al. 1999) or magnetars, and GWs excited in merging neutron star binaries and their possible effects on gamma ray bursts (GRBs).

In particular, we expect that the driving of MSW by gravitational waves can become interesting near stronglymagnetized compact sources of high-frequency GWs. The non-radial modes of relativistic stars produce GWs with frequencies of the order of $\mathrm{kHz}$. These can be excited in several ways: in the core-collapse leading to the formation of a neutron star, several modes of pulsation can be excited to large amplitudes. In newly-born neutron stars the quadrupole $f$ - and $r$-modes can become unstable to the emission of gravitational radiation, due to the CFS instability (see Stergioulas 1998; Andersson \& Kokkotas 2001 for recent reviews). In the merger of two neutron stars, if a black hole is not formed promptly, the resulting merged object will emit large amplitude GWs, due to the excitation of it's nonradial modes (see Shibata \& Uryu 2001). If strongly magnetized neutron stars (magnetars) exists, then the coupling of their secular spin-down with their very large magnetic field can lead to crust-fracturing and excitation of the quadrupole torsional mode in the crust of the star (see Duncan 1998). In all the above cases, highfrequency gravitational waves travel through the magnetosphere of the compact object.

In addition to GWs emitted by normal-mode excitation in neutron stars, the main frequency of GWs produced in the late stages of a neutron star binary inspiral is several hundred $\mathrm{Hz}$ to a few $\mathrm{kHz}$ (see e.g. Duez et al. 2000). In such a case, however, the geometry of the magnetic field changes rapidly and this has to be taken into account. 


\section{Summary and conclusion}

In this article we study for the first time the coupling of magnetosonic waves with GWs. Using the linearized general-relativistic hydromagnetic equations, we found the general dispersion relation for coupling of GW with the MHD waves. We showed that in the linear approximation only the fast magnetosonic waves will be coupled with the GW. We analyzed the linear dispersion relation for the GW-MSW coupling, and found the relation between the amplitudes of the two waves, when they are in resonance.

In carrying out this study we have made several simplification: (1) we treat only one polarization for the GW (see Eq. (4)), (2) the GW was treated as an external driver for the plasma, (3) we ignore non-linear terms and obliquely propagating waves. The above points, as well as the growth rate of driven MSW waves for specific GW sources, will be discussed in future work.

Acknowledgements. We thank Dr. K. Kokkotas for making several suggestion and keeping up with our work and Bernard Schutz, Joachim Moortgat and Gerard't Hooft for stimulating discussions. N. S. acknowledges the support by the European Union grant HPRN-CT-2000-00137.

\section{References}

Andersson, N., \& Kokkotas, K. D. 2001, Int. J. Mod. Phys. D., in press [gr-qc/0010102]

Andersson, N., Kokkotas, K. D., \& Schutz, B. F. 1999, ApJ, 510,846

Asseo, E., Gerbal, D., Heyvaerts, J., et al. 1976, Phys. Rev. D, 13,2724

Brodin, G., \& Marklund, M. 1999, Phys. Rev. Lett., 82, 3012

Brodin, G., Marklund, M., \& Servin, M. 2001, Phys. Rev. D, 63, 124003

Chesters, D. 1973, Phys. Rev. D., 7, 2863
Cooperstock, F. I. 1968, Ann. Phys., 47, 173

Demianski, M. 1985, Relativistic Astrophysics (Pergamon Press, Oxford, UK)

Denisov, V. I. 1978, Sov. Phys. JETP, 42, 209

Dermer, D. C., Miller, J. A., \& Li, H. 1996, ApJ, 456, 106

DeWitt, B. S., \& Breheme, R. W. 1960, Ann. Phys., 9, 220

Duez, M. D., Baumgarte, T. W., \& Shapiro, S. L. 2001, Phys. Rev. D, 63, 084030

Duncan, R. C. 1998, ApJ, 498, L45

Ellis, G. F. R., \& van Elst, H. 1998, Cosmological Models, in Cargese Summer School 1998 Proceedings, ed. M. LachiezeRey, in press

Esposito, P. 1971, ApJ, 165, 165

Gerlach, U. H. 1974, Phys. Rev. Lett., 32, 1023

Gertsenshtein, M. E. 1962, Sov. Phys. JETP, 14, 84

Grishchuk, L. P., \& Polnarev, A. G. 1976, in General Relativity and Gravitation, 2, ed. A. Held (Plenum Press)

Hoshino, M., Arons, J., Gallant, Y. A., \& Langdon, B. A. 1992, ApJ, 390, 454

Ignat'ev, Yu. G. 1997, Phys. Lett. A., 230, 171

Landau, L. D., \& Lifshitz, E. M. 1975, The Classical Theory of Fields (Pergamon Press, Oxford, UK)

Macdonald, D., \& Thorne, K. S. 1982, MNRAS, 198, 345

Macedo, P. G., \& Nelson, A. G. 1982, Phys. Rev. D., 28, 2382

Marklund, M., Brodin, G., \& Dunsby, P. K. S. 2000, ApJ, 236, 875

Papadopoulos, D., \& Esposito, F. P. 1982, ApJ, 257, 10

Papadopoulos, D., \& Esposito, F. P. 1985, ApJ, 292, 330

Polnarev, A. G. 1972, Sov. Phys. JEPT, 35, 834

Servin, M., Brodin, G., Bradley, M., et al. 2000, Phys. Rev. E, 62,8493

Servin, M., Brodin, G., \& Marklund, M. 2001 [gr-qc/0102031]

Shibata, M., \& Uryu, K. 2001 [astro-ph/0104409]

Stergioulas, N. 1998, Living Reviews in Relativity, 1998-8, www.livingreviews.org/Articles/Volume1/ 1998-8stergio/

Varvoglis, H., \& Papadopoulos, D. 1992, A\&A, 261, 664

Weber, J. 1960, Phys. Rev., 117, 306

Zel'dovich, Y. B. 1974, Sov. Phys. JETP, 38, 652 